\title{
ELCS in Ice: Cryo-electron Microscopy of Nuclear Envelope-Limited Chromatin Sheets
}

\author{
Mikhail Eltsov ${ }^{1}$, Sergey Sosnovski ${ }^{2}$ Ada L. Olins ${ }^{3}$ and Donald E. Olins ${ }^{3}$ \\ 1. Cell Biology and Biophysics Unit, European Molecular Biology Laboratory, Heidelberg, Germany \\ 2. Neurophysiology \& New Microscopies Laboratory, INSERM U603 - CNRS UMR 8154, Paris, France \\ ${ }^{3 .}$ University of New England, College of Pharmacy, Portland, Maine, USA
}

What is the structure of chromatin adjacent to the nuclear envelope (NE)? Does this surface chromatin exist in vivo as $\sim 10 \mathrm{~nm}$ (nucleosomal "beads on a string") or $\sim 30 \mathrm{~nm}$ (higher order polynucleosome folding)? Prior to the discovery of nucleosomes, employing aldehyde fixatives and thin-section electron microscopy on chicken erythrocyte nuclei, evidence was presented $[1,2]$ that condensed chromatin adjacent to the NE is organized in layers composed of $\sim 17 \mathrm{~nm}$ diameter "unit threads" with center-to-center spacings of $\sim 28 \mathrm{~nm}$, running parallel to the $\mathrm{NE}$, being especially clear in tangential sections. Subsequent studies with fish granulocytes [3] indentified extensions of the NE with a $\sim 30 \mathrm{~nm}$ thick chromatin layer, interpreted as parallel "unit threads" sandwiched between two apposed NE ("Nuclear Envelope-limited Chromatin Sheets"; denoted by us "ELCS"). In 1998, we demonstrated that nuclear lobulation and ELCS are induced in high yield by addition of retinoic acid (RA) to myeloid leukemic HL-60/S4 cells [4]. The NE of RA-treated HL-60/S4 cells exhibit major changes in composition, including a decrease in lamins $\mathrm{A} / \mathrm{C}$ and an increase in lamin $\mathrm{B}$ receptor (LBR) [5]. The increase in LBR is essential for the nuclear shape changes; a stable knockdown of LBR in RA-treated HL-60/S4 cells prevents both lobulation and ELCS formation [6].

We chose to examine the ultrastructure of ELCS by three different methods: 1) Aldehyde fixation/dehydration/plastic embedding/sectioning and staining (Chem Fix), Fig. 1. 2) High-pressure freezing/freeze substitution into plastic/sectioning and staining (FS). 3) High-pressure freezing/cryo-sectioning/cryo-electron microscopy (CEMOVIS) [7], Fig. 2. ELCS could be clearly visualized by all three methods and, consequently, must exist in vivo. The measured corrected mean separation between the apposed NE was $33.9 \mathrm{~nm}$ (Chem Fix), $33.5 \mathrm{~nm}$ (FS) and $57.4 \mathrm{~nm}$ (CEMOVIS). The "30 nm" fibers could only be observed following aldehyde fixation; none were seen after FS or CEMOVIS. Electron microscopic tomography of Chem Fix ELCS enabled examination of tangential views. Striking crisscrossing patterns of $\sim 10-30 \mathrm{~nm}$ chromatin fibers were observed running parallel to the nuclear envelope (Fig. 1). Based upon our images, models were devised to reconcile the concept of a "polymer melt" [8] (i.e., disordered, interdigitated $\sim 10 \mathrm{~nm}$ fibers) with two structural features of ELCS: 1) the uniformity of ELCS thickness, spanning the distance between apposed NEs; 2) the crisscrossing pattern of chromatin fibers seen in tangential views. One model ("Ordered Melt", Fig. 3) envisages parallel chromatin fibers at the NE crisscrossing when the NEs come face-to-face in the ELCS; the other model ("Disordered Melt") postulates that the pattern arises during the fixation and embedding of the ELCS. The uniformity of ELCS thickness is interpreted to be from the superpositioning of two layers of $\sim 10-30 \mathrm{~nm}$ chromatin fibers within the ELCS. Dehydration and embedding in the Chem Fix and FS procedures is believed to produce shrinkage, compared to the CEMOVIS prepared ELCS. It is suggested that the larger thickness of CEMOVIS ELCS reflects the irregular zigzag folding of the $\sim 10 \mathrm{~nm}$ chomatin fiber in the in vivo state. It is tempting to speculate that regions of chromatin "Ordered Melt" might be determined by patterns of inner nuclear membrane proteins, such as LBR [9]. 


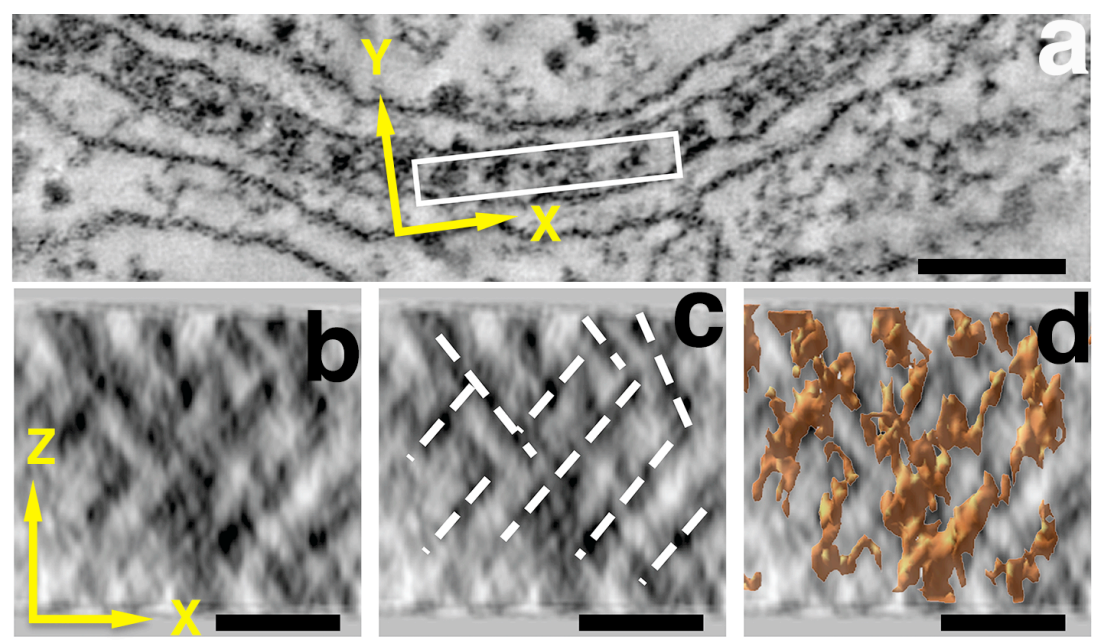

Fig. 1. Aldehyde fixed ELCS: a, Cross-section of "30 nm” fibers in rectangle. b, c, d, Rotated rectangle tangetial view; d, isosurface rendered. Bar in $\mathbf{a}, 100 \mathrm{~nm} ; \mathbf{b}, \mathbf{c}, \mathbf{d}, 50 \mathrm{~nm}$.

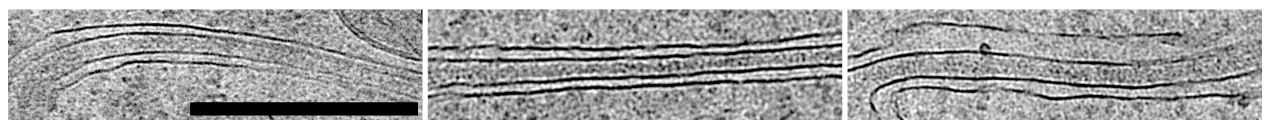

Fig. 2. Cryosections of ELCS: cross-section views with central chromatin. Bar, $500 \mathrm{~nm}$.

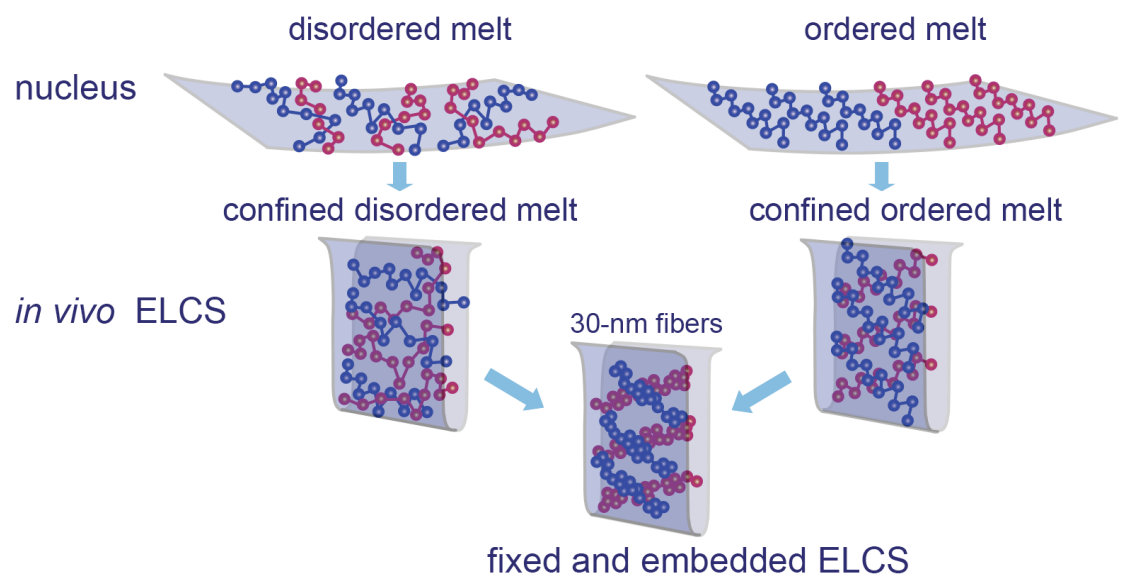

Fig. 3. Models for crisscross chromatin fiber formation in ELCS.

[1] Davies HG, et al., J Cell Sci, 16 (1974), p. 261-99.

[2] Everid AC, et al., J Cell Sci, 7 (1970), p. 35-48.

[3] Davies HG and Haynes ME, J Cell Sci, 17 (1975), p. 263-85.

[4] Olins AL, et al., Exp Cell Res, 245 (1998), p. 91-104.

[5] Olins AL, et al., Exp Cell Res, 268 (2001), p. 115-27.

[6] Olins AL, et al., Nucleus, 1 (2010), p. 506-12.

[7] Dubochet J, et al., Methods Cell Biol, 79 (2007), p. 385-406.

[8] Maeshima K, et al., Curr Opin Cell Biol, 22 (2010), p. 291-7.

[9] Thanks to EMBL, DKFZ and UNE for support; To P. Lichter, H. Herrmann and J. Langowski for opening their labs to ALO and DEO; To M. Morphew and A. Hoenger (Boulder CO) and R. Mellwig (EMBL). The Boulder Lab. for 3-D Electron Microscopy of Cells is supported by the NCRR, NIH. 